\title{
CONTINUOUS TRANSFORMATIONS AND STOCHASTIC DIFFERENTIAL EQUATIONS $\left(^{(}\right)$
}

\author{
BY \\ D. A. WOODWARD
}

Introduction. It is the purpose of this paper to construct stochastic processes $\{y(t), y(0)=0,0 \leqq t \leqq 1\}$ satisfying the differential equation

$$
d y(t)=f(y \mid t) d t+\sigma(t, y(t)) d x(t),
$$

where $\{x(t), x(0)=0,0 \leqq t \leqq 1\}$ is a Brownian motion process. Equation (0.1) has been studied by S. Bernstein [1], J. L. Doob [5] and others [2], [10]. In general, the solution given here is different from that given by these authors. Equation (0.1) is almost purely formal since the derivative $d x / d t$ fails to exist with probability one. In [2], [5], [10], the stochastic integral of $\mathrm{K}$. Ito [7], [8] is used to define an integrated form of $(0.1)$, which is solved as in [8] to obtain a transformation of sample functions. The present work involves a transformation of sample functions but the integral used is the functional integral of R. Cameron and R. Fagen [4]. E. B. Dynkin [6] has given a different method of attacking similar problems. Note that $f(y \mid t)$ is a functional.

1. Comparison with previous work. Let $m, n$, and $v$ be constants and specialize $(0.1)$ to

$$
d y(t)=m(y(t)+v) d t+n(y(t)+v) d x(t) .
$$

If the first derivatives of $y$ and $x$ are continuous, (1.1) is equivalent to

$$
y(t)=v\left(e^{m t+n x(t)}-1\right) .
$$

Equation (1.2) represents a transformation which is the unique continuous (in the uniform topology, say) extension of (1.2) to all continuous $x(t)$ with $x(0)=0$. The expected value of $y(t)$ as given by (1.2) is $v\left(m+n^{2} / 2\right) t$ $+o(t)$ as $t \rightarrow 0^{+}$, where the $\{x(t)\}$ process has variance parameter one. According to $[5$, p. 275], the expected value of $y(t)$ defined by (1.1) is $m v t+o(t)$ as $t \rightarrow 0^{+}$.

The backward and forward differencing schemes (8) and (8 bis) of $[1$, p. 12] suggest different stochastic differential equations but are equivalent. Following this method, the equation for $y(t)$ near $t=0$,

$$
y(t)=f(y \mid t / 2) t+\sigma(t / 2, y(t / 2)) x(t),
$$

may be obtained by neglecting terms of higher order than $t$ from equation (1.4) below.

Presented to the Society, January 23, 1964; received by the editors November 8, 1963.

$\left({ }^{1}\right)$ Work performed under the auspices of the U.S. Atomic Energy Commission. 
Notation. Subscripts on functions of more than one real variable denote partial derivatives. Thus $(\partial / \partial t) g(t, u)=g_{1}$ and $(\partial / \partial u) g(t, u)=g_{2}$.

$$
y(t)=f(y \mid 0) t+\sigma(0,0) \sigma_{2}(0,0) x(t / 2) x(t)+\sigma(0,0) x(t) .
$$

For $f=m(y(t)+v)$ and $\sigma=n(y(t)+v)$ the expected value of $y(t)$ as given by (1.4) is $m v t+n v n t / 2$, which agrees with that given by (1.2) as $t \rightarrow 0^{+}$.

The solution (1.2) of (1.1) was given by A. Rosenbloom [9].

2. Preliminary results. Let $C$ be the space of functions which are continuous on $[0,1]$ and vanish at zero. The topology on $C$ is that induced by the uniform norm denoted by $\|x(t)\|$. Let $C^{\prime}$ be the subspace of $C$ of functions which have continuous derivatives on $[0,1]$.

Definition. Let $U$ be an open subset of $C, F(x \mid t)$ be defined on $U \otimes$ $[0,1]$ and integrable in $t$ for each $x$, and $F(x \mid 1)$ be continuous on $U$. Define the functional integral of $F$ at $y$ on $[0, t]$ to be the following limit if it exists as a finite number:

$$
\lim _{x \rightarrow y, x \in C^{\prime}} \int_{0}^{t} F(x \mid s)(d x(s) / d s) d s=\int_{0}^{t} F(y \mid s) d^{*} y(s) .
$$

For $t=1$, the functional integral defined here is, if it exists, the same as that defined in [4] as can be seen from the proof of Theorem 1 there. The following theorem is similar to Theorem 2 of [4].

Theorem 1. Let $K$ be an open convex subset of $C, k \in K \cap C^{\prime}$, and $g(t, u)$ and $g_{1}(t, u)$ be continuous on $D=\{(t, u) \mid 0 \leqq t \leqq 1, u=y(t)$ for some $y \in K\}$, and if $(t, u),(t, v) \in D$, define

$$
G(t, u, v)=\int_{v}^{u} g(t, r) d r .
$$

Then if $y \in K$ the following functional integral exists and

$$
\begin{aligned}
\int_{0}^{t} g(s, y(s)) d^{*} y(s)= & G(t, y(t), k(t)) \\
& -\int_{0}^{t} G_{1}(s, y(s), k(s)) d s+\int_{0}^{t} g(s, k(s)) k^{\prime}(s) d s .
\end{aligned}
$$

Proof. The following argument depends only on the continuity of $G(t, u, k(t))$ on $D$. Let $\eta>0$ and $y_{0} \in K$ be given. The graph of $y_{0}(t)$ is compact, so an open covering of sets

$$
E(t)=\left\{\left(t^{\prime}, u\right)|| t-t^{\prime}|<\delta(t),| u-y_{0}(t) \mid<\delta(t)\right\},
$$

where $\delta(t)>0$ is so small that $\left(t^{\prime}, u\right) \in E(t)$ implies

$$
\left|G\left(t, y_{0}(t), k(t)\right)-G\left(t^{\prime}, u, k\left(t^{\prime}\right)\right)\right|<\eta,
$$

may be reduced to a finite covering $\left\{E\left(t_{k}\right)\right\}_{k=1, n}$. The union of this finite 
covering contains a strip of half-width $\delta>0$ about the graph of $y_{0}(t)$ such that $\left\|y(t)-y_{0}(t)\right\|<\delta$ implies

$$
\left\|G\left(t, y_{0}(t), k(t)\right)-G(t, y(t), k(t))\right\|<\eta .
$$

Thus $G(t, y(t), k(t))$ and $G_{1}(t, y(t), k(t))$ are continuous transformations in the uniform topologies. Equation (2.1) follows because its right-hand side with $y$ replaced by $x$ is $\int_{0}^{t} g(s, x(s)) d x(s)$ for $x \in C^{\prime} \cap K$. Since $C^{\prime}$ is dense in $C$ we have the

COROLLARY. Under the hypothesis of Theorem 1, the transformation $\int_{0}^{t} g(s, y(s)) d^{*} y(s)$ defined on $K$ into $C$ is the unique continuous extension of $\int_{0}^{t} g(s, y(s)) d y(s)$ defined on $K \cap C^{\prime}$.

Let $\sigma(t, u)$ be defined on an open set and

$$
\Omega_{0}=\{y \in C \mid \sigma(t, y(t))>0,0 \leqq t \leqq 1\} .
$$

THeOREM 2. If $\sigma(t, u)$ is defined and continuous on an open set, the set $\Omega_{0}$ is open and may be partitioned into at most a countable number of disjoint open convex components.

Proof. If $y_{0} \in \Omega_{0}, \sigma\left(t, y_{0}(t)\right)$ takes on its minimum value $v>0$. Use the argument in the proof of the theorem above, but choose $\delta(t)$ such that $\left(t^{\prime}, u\right) \in E(t)$ implies $\sigma\left(t^{\prime}, u\right)>v / 2$. It follows that $\Omega_{0}$ is open. Define the equivalence relation $y_{1} \sim y_{2}$ if and only if $y_{1}, y_{2} \in \Omega_{0}$ and $y \in C$ and

$$
\min \left(y_{1}(t), y_{2}(t)\right) \leqq y(t) \leqq \max \left(y_{1}(t), y_{2}(t)\right), \quad 0 \leqq t \leqq 1,
$$

imply $y \in \Omega_{0}$. The partition elements, called components, are disjoint and convex. The components are open since $\Omega_{0}$ is and members of a uniform sphere contained in $\Omega_{0}$ are equivalent. There are at most a countable number of components since $C$ has a countable dense subset.

3. Transformations. Let $\left\{K_{i}\right\}_{i=1, \infty}$ be the set of components of $\Omega_{0}$ according to Theorem 2. Let $k_{i} \in K_{i} \cap C^{\prime}, D_{i}=\{(t, u) \mid 0 \leqq t \leqq 1, u=y(t)$ for some $\left.y \in K_{i}\right\}, i=1, \infty$, and $G(t, u, v)=\int_{v}^{u} d r / \sigma(t, r)$ provided $(t, u),(t, v)$ $\in D_{i}$ for some $i, i=1, \infty$. The inverse function $H(t, w, v)$ such that $u$ $=H(t, w, v)$ and $w=G(t, u, v)$ is defined and continuous on

$$
\left\{(t, w, v) \mid(t, u),(t, v) \in D_{i}, w=G(t, u, v)\right\},
$$

$i=1, \infty$, by the implicit function theorem if $\sigma(t, u)$ is continuous. Define the transformations

$$
S_{i}: z(t)=G\left(t, y(t), k_{i}(t)\right)
$$

on $K_{i}, i=1, \infty$,

$$
T_{i}: x(t)=z(t)+\Lambda_{i}(z \mid t)
$$

on $S_{i}\left(K_{i}\right), i=1, \infty$, and

$$
R: x(t)=T_{i} S_{i} y \quad \text { on } K_{i}, i=1, \infty,
$$


on $\Omega_{0}$, where

$$
\Lambda_{i}(z \mid t)=M_{i}\left(H\left(\cdot, z(\cdot), k_{i}(\cdot)\right) \mid t\right)
$$

and

$$
M_{i}(y \mid t)=\int_{0}^{t}\left\{k_{i}^{\prime}(s) / \sigma\left(s, k_{i}(s)\right)-G_{1}\left(s, y(s), k_{i}(s)\right)-f(y \mid s) / \sigma(s, y(s))\right\} d s .
$$

Suppose $y \in \Omega_{0} \cap C^{\prime}$ and $\sigma(t, u)$ and $\sigma_{1}(t, u)$ are continuous. Then $x=R y$ implies $x \in C^{\prime}$ and $(0,1)$ holds. From the corollary to Theorem 1 we have the

Theorem 3. Let $\sigma(t, u)$ and $\sigma_{1}(t, u)$ be defined and continuous on the same open set and $f(y \mid t)$ be continuous on $\Omega_{0} \otimes[0,1]$. Then $(0.1)$ defines the unique continuous mapping $R$ on $\Omega_{0}$ into $C$.

Suppose $x \in C^{\prime}$ and $x=R y$ for $y \in K_{i}$. Then $G\left(t, y(t), k_{i}(t)\right) \in C^{\prime}$ and since $H$ has continuous partial derivatives, $y \in C^{\prime}$ and we have the

CoRollaRY. Under the hypothesis of Theorem 3 , the pre-image of $C^{\prime} \cap R\left(\Omega_{0}\right)$ is $C^{\prime} \cap \Omega_{0}$.

Let $\Omega_{1}$ be the set of all extended real-valued functions on $(0,1]$ which are not members of $\Omega_{0}$. In this section $\Omega_{1}$ is used only to normalize the measure on the $\{y(t)\}$ process. For example, the infinite-valued solutions of

$$
d y(t)=-y^{2}(t) d t+d x(t)
$$

are members of $\Omega_{1}$ which then has positive measure as shown in [11]. Let $S_{y}$ be the $\sigma$-ring generated by $\Omega_{1}$ and the Borel subsets of $\Omega_{0}$. Let $R\left(\Omega_{1}\right)$ be the set of all extended real-valued functions on $(0,1]$ which are not members of $R\left(\Omega_{0}\right)$ and $\mathrm{S}_{x}$ be the $\sigma$-ring generated by $R\left(\Omega_{1}\right)$ and the Borel subsets of $R\left(\Omega_{0}\right)$.

Lemma 7 and Theorem 4 of [4] are stated here in the slightly weaker form of

Theorem A. Let the transformation

$$
T:(t)=z(t)+\Lambda(z \mid t)
$$

defined on the open subset $\Gamma$ of $C$ satisfy the following conditions.

(A1) On a uniform neighborhood $U_{0}$ of each $z_{0} \in \Gamma$ let

$$
\left.\frac{\partial}{\partial v} \Lambda(z+v r \mid t)\right|_{v=0}=\int_{0}^{1} N(z \mid t, s) r(s) d s
$$

if $(z, t, r) \in U_{0} \otimes[0,1] \otimes C$, where

$$
N(z \mid t, s)= \begin{cases}N^{1}(z \mid t, s), & 0 \leqq t<s \leqq 1 \\ (1 / 2) N^{1}(z \mid t, s)+(1 / 2) N^{2}(z \mid t, s), & 0 \leqq t=s \leqq 1 \\ N^{2}(z \mid t, s), & 0 \leqq s<t \leqq 1\end{cases}
$$


and $N^{1}$ and $N^{2}$ are continuous on $U_{0} \otimes\{0 \leqq t \leqq s \leqq 1\}$ and $U_{0} \otimes\{0 \leqq s$ $\leqq t \leqq 1\}$, respectively.

Let $N(z \mid t, s)$ be bounded on $U_{0} \otimes[0,1] \otimes(0,1]$.

Let $D(z) \neq 0$ on $\Gamma$ where $D(z)$ is the Fredholm determinant,

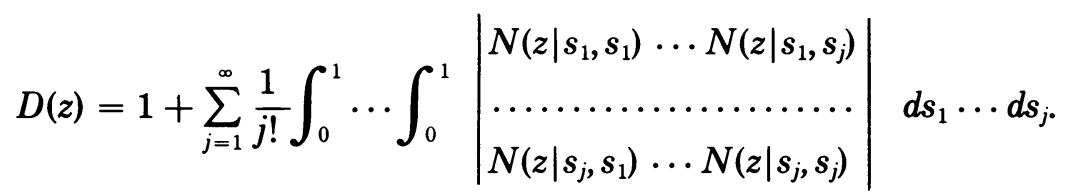

(A2) Let $N(z \mid 0, s)=0$ for $(z, s) \in \Gamma \otimes(0,1]$. Let $\Lambda(z \mid t) \in C$ when $z \in$ $\Gamma$ and $\partial \Lambda(z \mid t) / \partial t$ be continuous on $\Gamma \otimes[0,1]$.

Then $T$ is open. Moreover, suppose the following conditions hold.

(A3) On a uniform neighborhood $U_{0}$ of each $z_{0} \in \Gamma$ let, for each fixed $t$ $\in[0,1]$,

$$
\int_{0}^{1}\left(N\left(z_{1} \mid t, s\right)-N\left(z_{2} \mid t, s\right)\right)^{2} d s \rightarrow 0
$$

uniformly in $z_{1}, z_{2} \in U_{0}$ as $\left\|z_{1}(t)-z_{2}(t)\right\| \rightarrow 0$.

(A4) On a uniform neighborhood $U_{0}$ of each $z_{0} \in \Gamma$ let

$$
\int_{0}^{1}\left[(\partial / \partial t)(1 / \epsilon) \int_{t}^{t+\epsilon} \Lambda\left((1 / \epsilon) \int_{(\cdot)-\epsilon}^{(\cdot)} z(u) d u \mid s\right) d s\right] d z(t),
$$

where functions of a real variable are assumed to be defined continuously outside the interval $(0,1)$, be defined and bounded below in $(x, \epsilon)$ on $U_{0} \otimes\left(0, \epsilon_{0}\right)$ for some positive $\epsilon_{0}<(1 / 2)$.

(A5) $\int_{0}^{1}(\partial / \partial t) \Lambda(z \mid t) d^{*} z(t)$ exists as defined in $\$ 2$ for all $z \in \Gamma$.

(A6) $T$ is one-to-one on $\Gamma$.

Then if $F$ is a Wiener measurable functional such that either of the following Wiener integrals (expectations on the Brownian motion process with variance parameter (1/2)) exists, they both exist and are equal.

$$
\int_{T_{\Gamma}}^{w} F(x) d_{w} x=\int_{\Gamma}^{w} F(T z) J_{T}(z) d_{w} z
$$

where

$$
J_{T}(z)=|D(z)| \exp \left\{-2 \int_{0}^{1}(\partial / \partial t) \Lambda(z \mid t) d^{*} z(t)-\int_{0}^{1}[(\partial / \partial t) \Lambda(z \mid t)]^{2} d t\right\} .
$$

An application of Theorem $\mathrm{A}$ is our main result.

Theorem 4. Let $\sigma(t, u)$ and $\sigma_{1}(t, u)$ be defined and continuous on the same open set and $f(y \mid t)$ be continuous on $\Omega_{0} \otimes[0,1]$. Let the solutions $y \in C^{\prime}$ $\cap \Omega_{0}$ of $(0.1)$ be unique for each $x \in C^{\prime}$. For each $i, i=1, \infty$, let $T_{i}$ defined on $S_{i}\left(K_{i}\right)$ satisfy (A1). Then $R$ is one-to-one, open, and continuous. Thus a measure on one of the $\sigma$-rings, $\mathbf{S}_{x}$ and $\mathbf{S}_{y}$, and $R$ define a measure on the 
other with the same total measure. Suppose the measure on $\mathbf{S}_{x}$ is that of the restriction of the Brownian motion process with variance parameter (1/2) and the measure defined by $R^{-1}$ on $\mathbf{S}_{y}$ is denoted by $P_{y}$. Suppose also that $T_{i}$ satisfies conditions (A3) and (A5) on $S_{i}\left(K_{i}\right), i=1, \infty$. Then if $F$ is a measurable functional on $\Omega_{0}$ such that either side of (3.4) exists, (3.4) holds.

$$
\int_{\Omega_{0}} F(y) d P_{y}=\sum_{i=1}^{\infty} \int_{S_{i}\left(K_{i}\right)}^{w} F\left(H\left(\cdot, z(\cdot), k_{i}(\cdot)\right)\right) J_{T_{i}}(z) d_{w} z,
$$

where $J_{T_{i}}$ is given by (3.3) and the Wiener integral notation is used on the right.

Proof. For $i=1, \infty, T_{i}$ satisfies (A1) by hypothesis and (A2) because of the form of $\Lambda_{i}$. From the first part of Theorem A, $T_{i}$ is open, $i=1, \infty$. Since $S_{i}$ is open, $i=1, \infty, R$ is open. The uniqueness of solutions $y \in C^{\prime}$ $\cap \Omega_{0}$ of $(0.1)$ for $x \in C^{\prime}$, openness of $R$, and the corollary to Theorem 3 imply that $R$ is one-to-one. The continuity of $R$ follows from Theorem 3 . Now assume all the hypothesis of Theorem 4. Since $F$ is Borel measurable and $R$ is open, $F\left(R^{-1} x\right)$ is Wiener measurable on $R\left(K_{i}\right), i=1, \infty$. For $i$ $=1, \infty, T_{i}$ satisfies (A6) because $T_{i}=R S_{i}^{-1}$. From the definition of the functional integral used here, for each $i=1, \infty$ and $z_{0} \in S_{i}\left(K_{i}\right)$ there exists a uniform sphere of radius $r>0$ about $z_{0}$ such that $\int_{0}^{1}(\partial / \partial t) \Lambda_{i}(z \mid t)(d z(t) / d t) d t$ is bounded for $z \in C^{\prime} \cap V$. Choose $\epsilon_{0}>0$ such that $\epsilon<\epsilon_{0}$ implies

$$
\left\|\int_{\min (0, t-\epsilon)}^{t}(1 / \epsilon)\left(z_{0}(s)-z_{0}(t)\right) d s\right\|<r / 2 \text {. }
$$

Then (A4) is satisfied on $V_{1} \otimes\left(0, \epsilon_{0}\right)$ where $V_{1}$ is the uniform sphere of radius $r / 2$ about $z_{0}$. From Theorem $A$ the right-hand side of (3.4) is

$$
\sum_{i=1}^{\infty} \int_{T_{i} S_{i}\left(K_{i}\right)}^{w} F\left(S_{i}^{-1} T_{i}^{-1} x\right) d_{w} x=\int_{R\left(\Omega_{0}\right)}^{w} F\left(R^{-1} x\right) d_{w} x .
$$

Equation (3.4) follows by definition.

As an example, the probability of finite continuous solutions $y(t)$ of (3.1) is shown in [3] to be

$$
\int_{C}^{w} \exp \left\{\int_{0}^{1}\left(x(t)-(x(t))^{4}\right) d t-2(x(1))^{3} / 3\right\} d_{w} x .
$$

4. Generalizations. A formal solution of (1.1) is

$$
y(t)=e^{m t+n x(t)}\left\{\int_{0}^{t} e^{-m s-n x(s)} n v d^{*} x(s)+\int_{0}^{t} e^{-m s-n x(s)} m v d s\right\} .
$$

That (4.1) is the same as (1.2) may be seen either from Theorem 1 or its corollary. Stochastic differential equations of more general type than (0.1) may be solved by using standard integration techniques. For example, if the total differential equation $P d y+Q d t+R d x=0$ is complete, then the transformation $x(t) \rightarrow y(t)$ is implicit. On the other hand (1.4) suggests a new type of "differential" equation. 
The theory of $\$ 3$ does not apply to the equation

$$
d y(t)=-\ldots h(t) d t+n y(t) d x(t)
$$

since $\sigma(0, y(0))=n y(0)=0$. A solution of $(4.2)$ is

$$
y(t)=e^{n x(t)} \int_{0}^{t} h(s) e^{-n x(s)} d s .
$$

In this case $y^{\prime}(0)=h(0)$ with probability one if $h$ is continuous.

In $\$ 3$ the transformation $R$ is factored into $T_{i} S_{i}$ on $K_{i}$. In place of $S_{i}$ one might wish to use

$$
S_{i}^{\prime}: v(t)=\int_{0}^{t} d^{*} y(s) / \sigma(s, y(s))=V_{i} S_{i} y,
$$

where $V_{i}$ is the transformation

$$
V_{i}: v(t)=z(t)+\int_{0}^{t}\left\{k_{i}(s) / \sigma\left(s, k_{i}(s)\right)-G_{1}\left(s, H\left(s, z(s), k_{i}(s)\right), k_{i}(s)\right)\right\} d s,
$$

which is of Volterra type and consequently one-to-one. Similarly, if $\sigma$ is a functional $\sigma(y \mid t)$, such that the transformation

$$
S_{i}^{\prime}: v(t)=\int_{0}^{t} d^{*} y(s) / \sigma(y \mid s)
$$

is one-to-one, open, and continuous, then the more general problem could be treated. An interesting formula in this connection may be found in $\$ 8$ of $[3]$.

\section{REFERENCES}

1. S Bernstein, Equations differentielles stochastiques, Actualités Sci. Ind. No. 138, Hermann, Paris, 1938; pp. 5-31.

2. A. V. Skorokhod, Stochastic equations for diffusion processes in a bounded region, Theor. Probability Appl. 6 (1961), 264-274.

3. R. H. Cameron, Nonlinear Volterra functional equations and linear parabolic differential systems, J. Analyse Math. 5 (1956/57), 136-182.

4. R. H. Cameron and R. E. Fagen, Nonlinear transformations of Volterra type in Wiener space, Trans. Amer. Math. Soc. 75 (1953), 552-575.

5. J. L. Doob, Stochastic processes, Wiley, New York, 1953.

6. E. B. Dynkin, Transformations of Markov processes connected with additive functionals, Proc. Fourth Berkeley Sympos. Math. Statist. and Prob., Vol. II, pp. 117-142, California Univ. Press, Berkeley, Calif., 1961.

7. Kiyosi Ito, Stochastic integral, Proc. Imperial Acad. Tokyo 20 (1944), 519-524.

8.

9. A. Rosenbloom, Analysis of linear systems with randomly time-varying parameters, Proc. Sympos. Information Networks, Polytechnic Institute of Brooklyn, New York, 1955.

10. N. Takeaki, Stability problems of random linear systems of the first order, Mem. Fac. Sci. Kyushu Univ. Ser. A 16 (1962), 47-59.

11. D. A. Woodward, On a special integral equation, Proc. Amer. Math. Soc. 10 (1959), 853-854.

Argonne National Laboratory, Argonne, ILLINOIS 